\title{
Stellar populations in normal galaxies
}

\author{
Gustavo Bruzual A. ${ }^{1}$ \\ ${ }^{1}$ CIDA, Apartado Postal 264, Mérida, Venezuela email: bruzual@cida.ve
}

\begin{abstract}
I describe very briefly the new libraries of empirical spectra of stars covering wide ranges of values of the atmospheric parameters $\mathrm{T}_{\text {eff }}, \log \mathrm{g},[\mathrm{Fe} / \mathrm{H}]$, as well as spectral type, that have become available in the recent past, among them the HNGSL, MILES, UVES-POP, and Indo-US libraries. I show the results of using the HNGSL to build population synthesis models. These libraries are complementary in spectral resolution and wavelength coverage, and will prove extremely useful to describe spectral features expected in galaxy spectra from the NUV to the NIR.
\end{abstract}

\section{Introduction}

In a recent paper Bruzual \& Charlot (2003), hereafter BC03, have examined in detail the advantages of using intermediate resolution stellar spectra in population synthesis and galaxy evolution models. In these models BC03 use the STELIB library compiled by Le Borgne et al. (2003). This library contains observed spectra of 249 stars in a wide range of metallicities in the wavelength range from $3200 \AA$ to $9500 \AA$ at a resolution of $3 \AA \mathrm{FWHM}$ (corresponding to a median resolving power of $\lambda / \Delta \lambda \approx 2000$ ), with a sampling interval of $1 \AA$ and a signal-to-noise ratio of typically 50 per pixel. The BC03 models reproduce in detail typical galaxy spectra extracted from the SDSS Early Data Release (Stoughton et al. 2002). From these spectral fits one can constrain physical parameters such as the star formation history, metallicity, and dust content of galaxies (Heavens et al. 2004; Mateu et al. 2004. See also several papers in this conference, e.g. Cid-Fernández et al.). The medium resolution BC03 models enable accurate studies of absorption-line strengths in galaxies containing stars over the full range of ages and can reproduce simultaneously the observed strengths of those Lick indices that do not depend strongly on elemental abundance ratios, provided that the observed velocity dispersion of the galaxies is accounted for properly, and offer the possibility to explore new indices over the full optical range of the STELIB atlas, i.e. $3200 \AA$ to $9500 \AA$. To extend the spectral coverage of the models beyond these limits, we must recur to other libraries. For solar metallicity models, the Pickles (1998) library can be used to extend the STELIB spectral coverage down to $1150 \AA$ in the UV end and up to $2.5 \mu \mathrm{m}$ at the red end, with a sampling interval of $5 \AA$ pixel $^{-1}$ and a median resolving power of $(\lambda / \Delta \lambda \approx 500)$. The UV spectra in the Pickles (1998) atlas are based on IUE spectra of bright stars. At all metallicities the BC03 models are extended down to $91 \AA$ in the UV side and up to $160 \mu \mathrm{m}$ at the other end using the theoretical model atmospheres included in the BaSeL series of libraries compiled by Lejeune et al. (1997), Lejeune et al. (1998), and Westera et al. (2002), but at a resolving power considerably lower than for STELIB $(\lambda / \Delta \lambda \approx 200-500)$. Given the success in reproducing observed galaxy spectra with the synthesis models built with STELIB, it is important to improve: first, the spectral resolution outside the STELIB range, and second, the coverage of the HRD by including more spectral types than those available in STELIB. These goals are possible now thanks to several compilations of stellar spectra that have become available in the recent past. In this paper I describe briefly these libraries and show examples of model galaxy spectra 
built using one of these libraries. The full implementation of the new libraries in the population synthesis models is in preparation by Bruzual \& Charlot.

\section{New libraries}

A large number (4!) of libraries containing medium to high spectral resolution observed spectra of excellent quality for large numbers (hundreds to thousand!) of stars have become available during the last few months. One of the main objectives of the observers who invested large amounts of time and effort assembling these data sets is to build libraries suitable for population synthesis. In this respect the stars in the libraries have been selected to provide broad coverage of the atmospheric parameters $\mathrm{T}_{\text {eff }}, \log \mathrm{g}$, $[\mathrm{Fe} / \mathrm{H}]$, as well as spectral type, throughout the HRD. Below I describe very briefly the main characteristics of each of these libraries, especially those which are relevant for population synthesis models.

\section{1. $H N G S L$}

The Hubble's New Generation Spectral Library (Heap \& Lanz 2003) contains spectra for over 200 stars (this number will increase in the future) whose fundamental parameters, including chemical abundance, are well known from careful analysis of the visual spectrum. The spectra cover fully the wavelength range from $1700 \AA$ to $10,200 \AA$. The advantage of this library over the ones listed below is the excellent coverage of the nearUV and the range from $9000 \AA$ to $10,200 \AA$, which is generally noisy or absent in the other data sets.

\subsection{MILES}

The Medium resolution INT Library of Empirical Spectra (Sánchez-Blázquez et al. 2003), contains carefully calibrated and homogeneous quality spectra for 1003 stars in the wavelength range $3500 \AA$ to $7500 \AA$ with $2 \AA$ spectral resolution and dispersion $0.9 \AA$ pixel ${ }^{-1}$. The stars included in this library were chosen aiming at sampling stellar atmospheric parameters as completely as possible.

\subsection{UVES POP Library}

The UVES Paranal Observatory Project (Bagnulo et al. 2004), has produced a library of high resolution $(\lambda / \Delta \lambda \approx 80,000)$ and high signal-to-noise ratio spectra for over 400 stars distributed throughout the HRD. For most of the spectra, the typical final SNR obtained in the $\mathrm{V}$ band is between 300 and 500. The UVES POP library is the richest available database of observed optical spectral lines.

\subsection{Indo-US library}

The Indo-US library (Valdes et al. 2004) contains complete spectra over the entire $3460 \AA$ to $9464 \AA$ wavelength region for 885 stars obtained with the $0.9 \mathrm{~m}$ Coudé Feed telescope at KPNO. The spectral resolution is $\approx 1 \AA$ and the dispersion $0.44 \AA$ pixel $^{-1}$. The library includes data for an additional 388 stars, but only with partial spectral coverage.

\subsection{High-spectral resolution theoretical libraries}

There are several on-going efforts to improve the existing grids of theoretical model atmospheres including the computation of high resolution theoretical spectra for stars whose physical parameters are of interest for population synthesis. See, for example, Coelho et al. (2003), Bertone et al. (2004), Murphy \& Meiksin (2004), Peterson et al. (2004). 
1 Gyr, $Z_{\odot}$, Chabrier IMF, Padova 1994

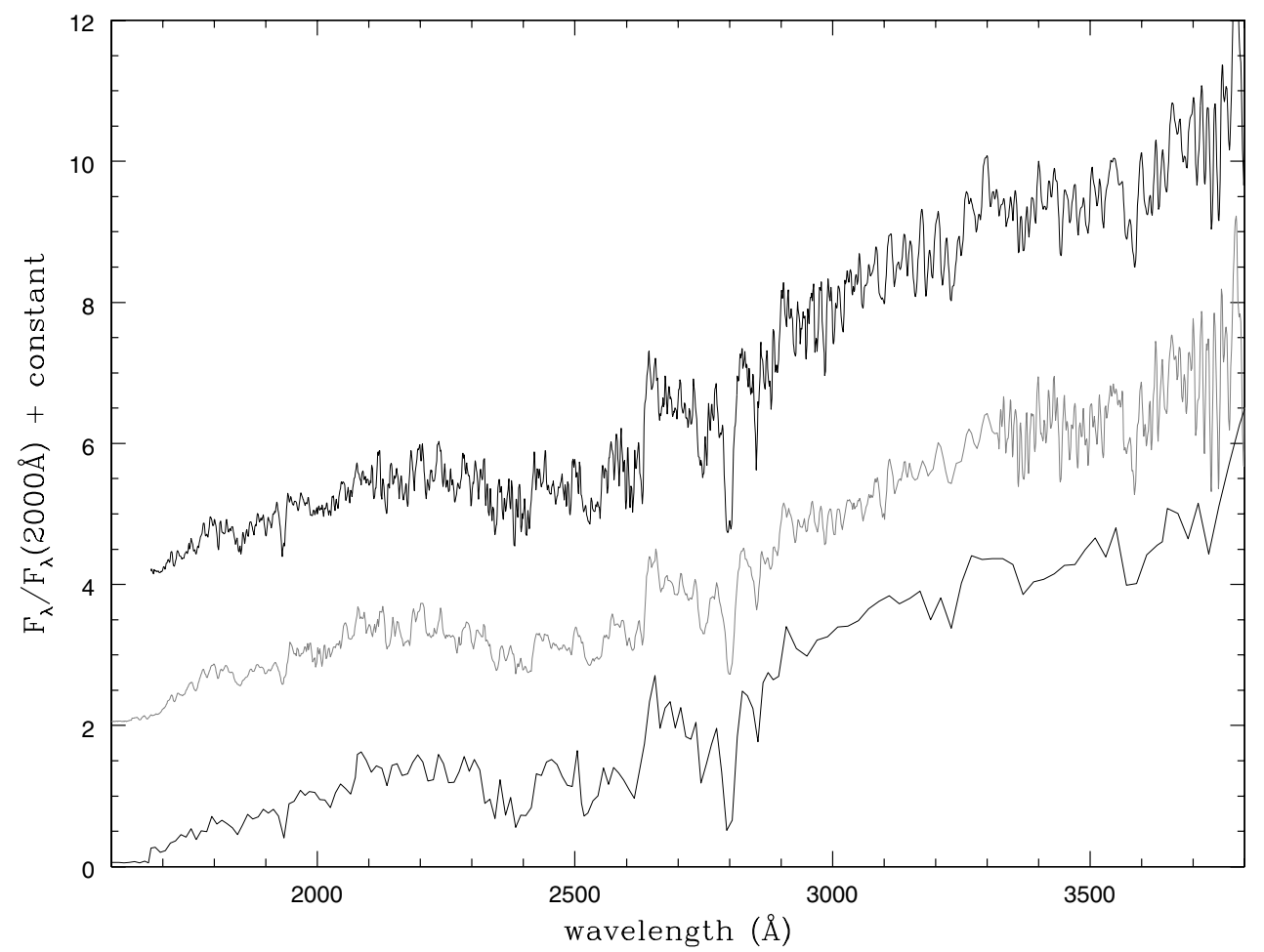

Figure 1. BC03 standard SSP model spectra for solar metallicity at 1 Gyr in the wavelength range from $1600 \AA$ to $3800 \AA$. The spectrum on top (thick black line) is built using the HNGSL, the one in the middle (gray line) uses the Pickles library and STELIB, and the bottom one (thin black line) the BaSel 3.1 library. The spectra have been normalized at $2000 \AA$ and shifted arbitrarily in the vertical direction for clarity.

\section{Results}

In this section I show sample spectra from the 'standard' reference model defined by BC03. This model represents a simple stellar population (SSP) computed using the Padova 1994 evolutionary tracks, the STELIB/Pickles/BaSeL 3.1 spectral libraries and the Chabrier (2003) IMF truncated at 0.1 and $100 \mathrm{M}_{\odot}$ (see BC03 for details). For illustration purposes I have computed a similar model but using the HNGSL (Heap \& Lanz 2003) instead of STELIB to represent the stellar spectra.

Figures 1 and 2 show clearly the advantages of using the HNGSL to study the near UV below $3300 \AA$. Spectral features, lines and discontinuities, are much better defined in the HNGSL spectra than in the IUE (used in the Pickles library) and the BaSeL 3.1 library. However, the colors measured from the continuum in these spectra produce very similar values. The higher spectral resolution of STELIB above $3300 \AA$ compared to HNGSL is clearly seen in these figures.

In Figure 3 I compare the predicted spectra in the optical range for a very young (500 Myr) stellar population of solar metallicity using the indicated spectral libraries. Despite the very similar continuum, the higher resolution STELIB model is to be preferred in this optical range to study spectral lines. Figures 1 to 3 show clearly that the BaSeL 3.1 
12 Gyr, $\mathrm{Z}_{\odot}$, Chabrier IMF, Padova 1994

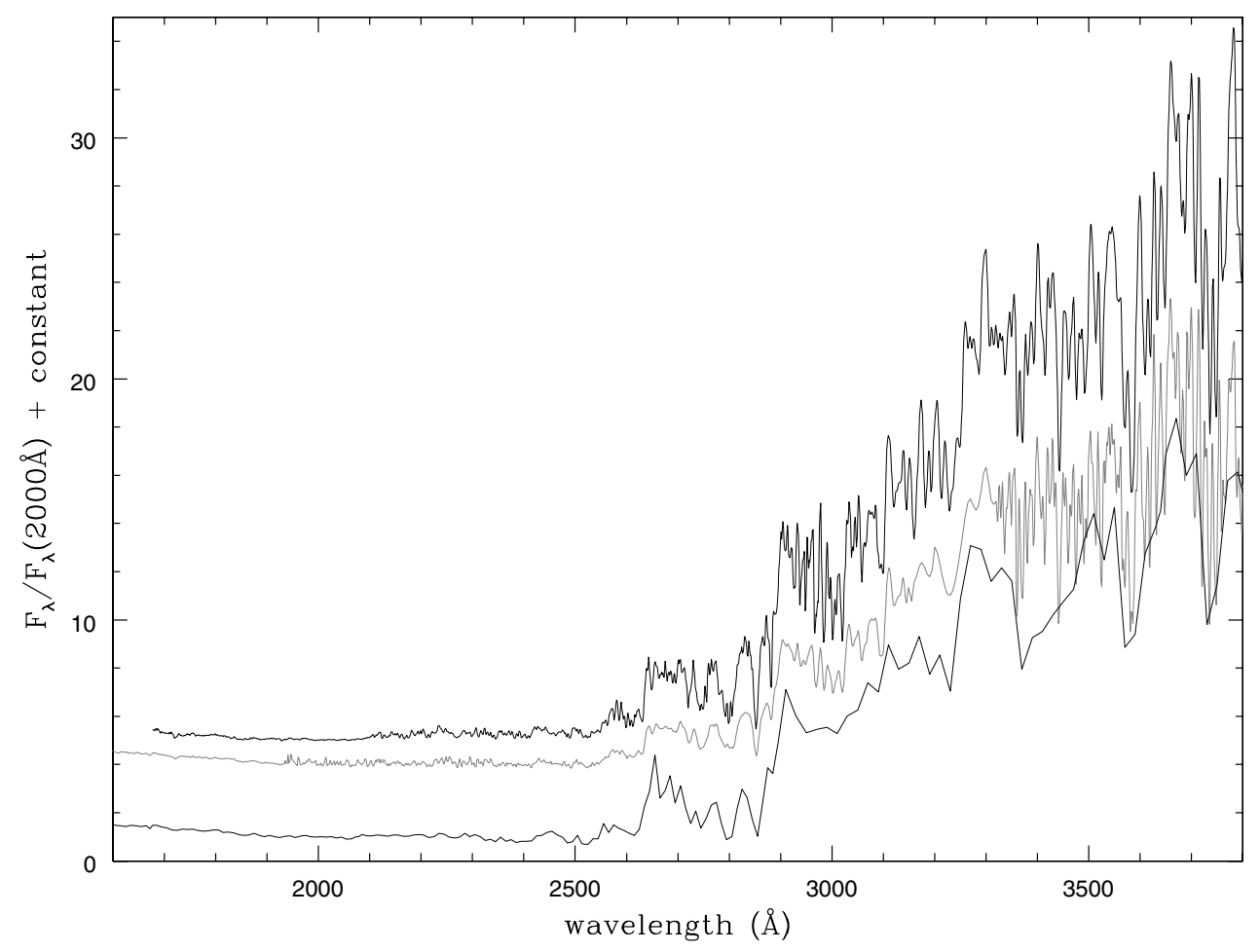

Figure 2. BC03 standard SSP model spectra for solar metallicity at 12 Gyr in the wavelength range from $1600 \AA$ to $3800 \AA$. The spectrum on top (thick black line) is built using the HNGSL, the one in the middle (gray line) uses the Pickles library and STELIB, and the bottom one (thin black line) the BaSel 3.1 library. The spectra have been normalized at $2000 \AA$ and shifted arbitrarily in the vertical direction for clarity.

models are not adequate at all to study spectral features, even though they may provide a good definition of the continuum level.

The spectra in Figure 4 are useful to study the different behavior of models computed with different libraries in the region around and above $9000 \AA$. There is a clear difference in the continuum level predicted by the HNGSL and STELIB models (lowest level) compared to the level predicted by the BaSeL 3.1 and the Pickles library models (highest level). The HNGSL model is to be preferred, given the higher SNR of this spectrum in this region. However, spectral features below $8500 \AA$ are more clearly seen in the higher resolution STELIB model.

\section{Conclusions}

New libraries of empirical spectra of stars covering wide ranges of values of the atmospheric parameters $\mathrm{T}_{\text {eff }}, \log \mathrm{g},[\mathrm{Fe} / \mathrm{H}]$, as well as spectral type, that have become available recently, are complementary in spectral resolution and wavelength coverage. Preliminary models built using the HNGSL show that this sort of library will prove extremely useful to describe spectral features expected in galaxy spectra of various ages and metallicities from the NUV to the NIR. Due to the lack of space I have compared previous models with new models built only with the HNGSL. Models that use all the 
500 Myr, $\mathrm{Z}_{\odot}$, Chabrier IMF, Padova 1994

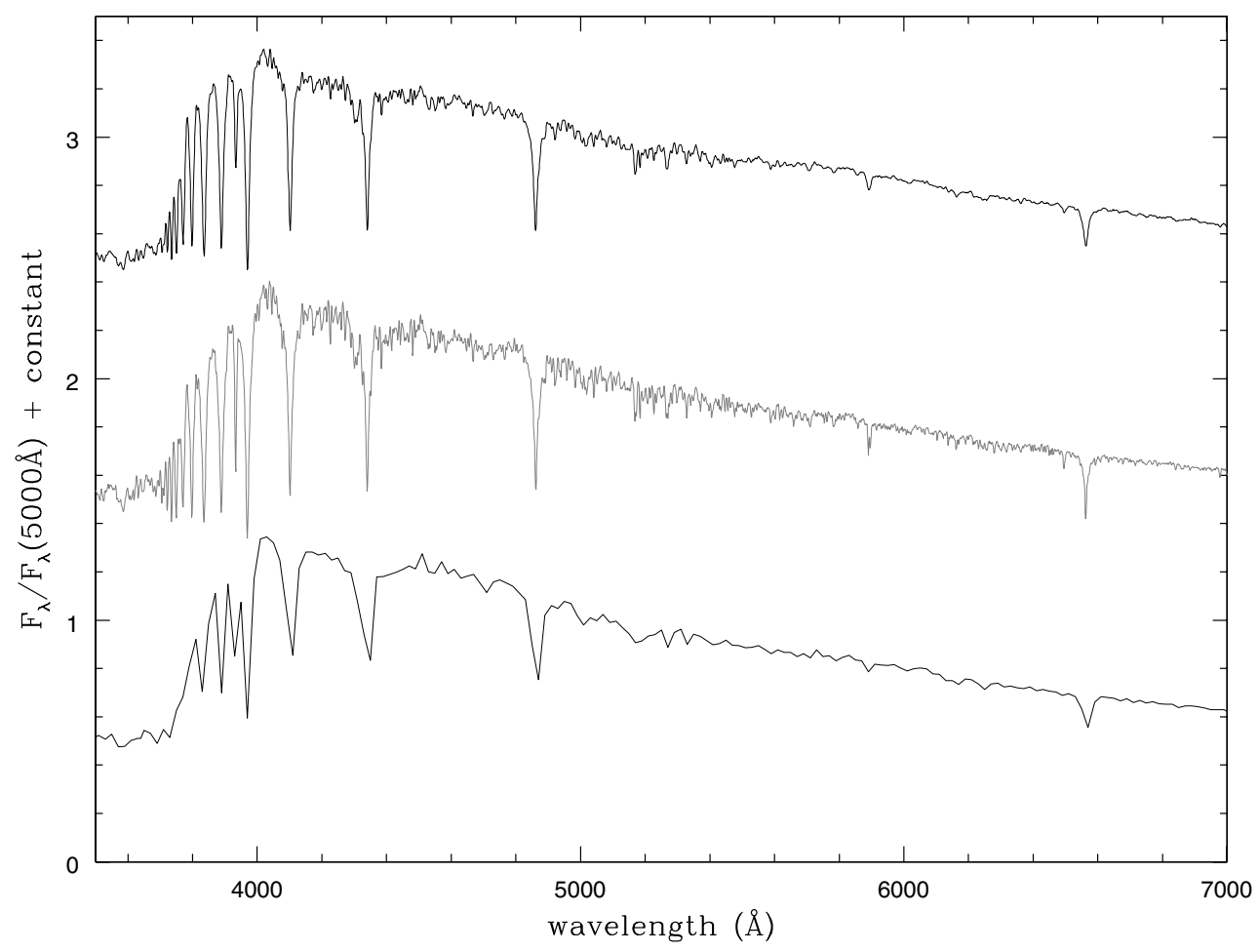

Figure 3. BC03 standard SSP model spectra for solar metallicity at $500 \mathrm{Myr}$ in the wavelength range from $3500 \AA$ to $7000 \AA$. The spectrum on top (thick black line) is built using the HNGSL, the one in the middle (gray line) uses STELIB, and the bottom one (thin black line) the BaSel 3.1 library. The spectra have been normalized at $5000 \AA$ and shifted arbitrarily in the vertical direction for clarity.

libraries mentioned in $\S 2$ are being built and will be discussed in a forthcoming paper by Bruzual \& Charlot.

\section{References}

Bagnulo, S., Jehin, E., Ledoux, C., Cabanac, R., Melo, C., \& Gilmozzi, R. and the ESO Paranal Science Operations Team 2003, The Messenger, 114, 10

Bertone, E., Buzzoni, A., Rodríguez-Merino, L. H., \& Chávez, M. 2004, MmSAI, 75, 158

Bruzual A., G., \& Charlot, S. 2003, MNRAS, 344, 1000

Chabrier, G. 2003, PASP, 115, 763

Coelho, P., Barbuy, B., Melendez, J., Allen, D. M., \& Castilho, B. 2003, BASBr, 23, 98

Heap, S. R., \& Lanz, T. 2003, in Proceedings of the ESO-USM-MPE Workshop on Multiwavelength Mapping of Galaxy Formation and Evolution, Venice, (ed. A. Renzini), in press

Heavens, A., Panter, B., Jiménez, R., \& Dunlop, J. 2004, Nature, 428, 625

Le Borgne, J.-F., et al. 2003, A\&A, 402, 433

Lejeune, T., Cuisinier, F., \& Buser, R. 1997, A\&AS, 125, 229

Lejeune, T., Cuisinier, F., \& Buser, R. 1998, A\&AS, 130, 65

Mateu, J., Bruzual A., G., \& Magris, C., G. 2004, MNRAS, in preparation

Murphy, T., \& Meiksin, A. 2004, (astro-ph/0404010)

Peterson, R. C., et al. 2004, AAS, 204, 07.08 
12 Gyr, $\mathrm{Z}_{\odot}$, Chabrier IMF, Padova 1994

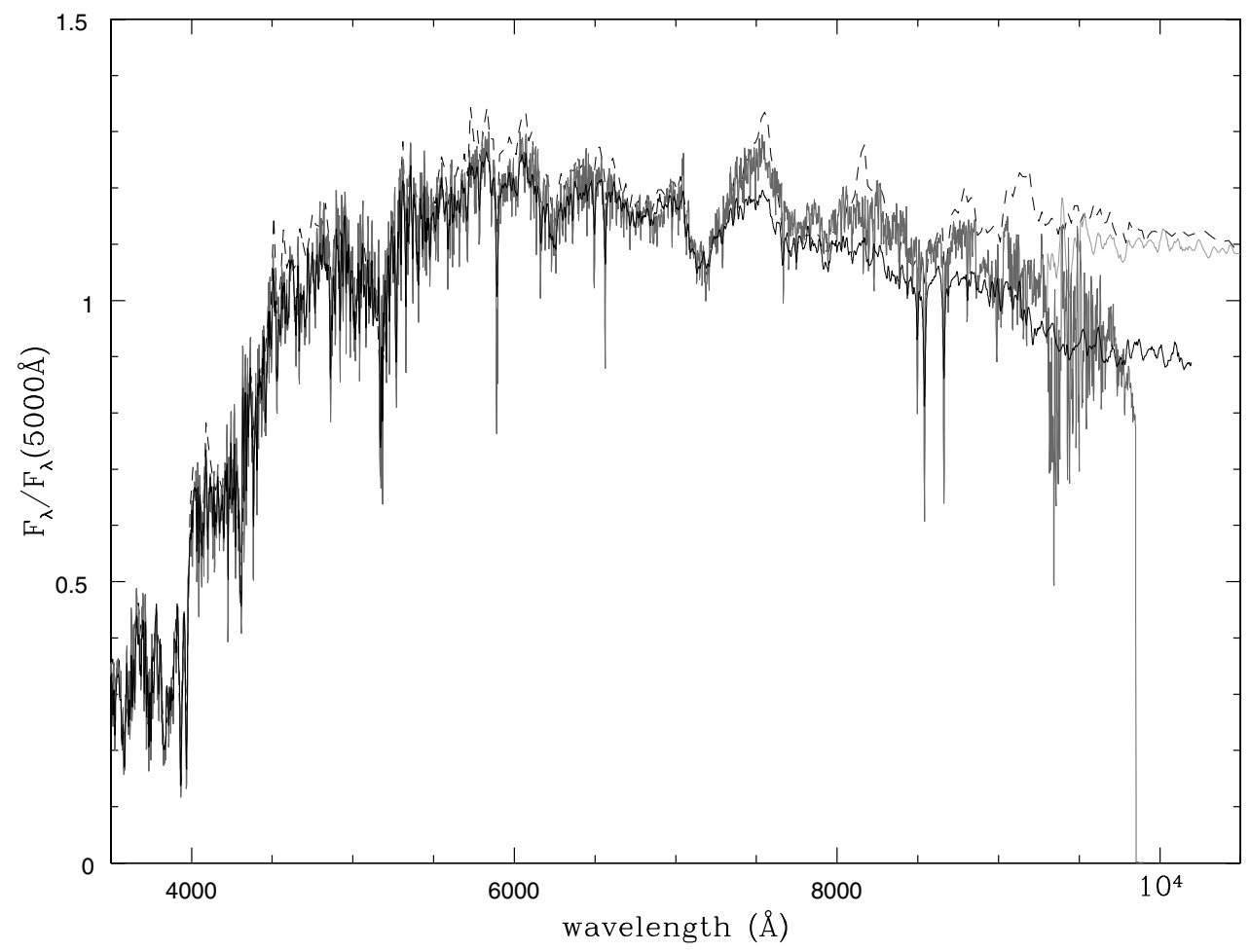

Figure 4. BC03 standard SSP model spectra for solar metallicity at 12 Gyr in the wavelength range from $3500 \AA$ to $10,500 \AA$. The black solid line (reaching up to $10,200 \AA$ ) represents the spectrum built using the HNGSL, the dark gray solid line (noisy above $9000 \AA$ ) uses STELIB, the light gray solid line uses STELIB up to $9000 \AA$ and the Pickles library at longer wavelengths, the black dashed line uses the BaSel 3.1 library. The spectra have been normalized at $5000 \AA$.

Pickles, A. J. 1998, PASP, 110, 863

Sánchez-Blázquez, P., et al. 2003, Rev. Mex. Astron. Astrofis. Conf. Ser., 17, 192

Stoughton, C., et al. 2002, AJ, 123, 485

Valdes, F., Gupta, R., Rose, J. A., Singh, H. P., \& Bell, D. J. 2004, ApJS, 152, 251

Westera, P., Lejeune, T., Buser, R., Cuisinier, F., \& Bruzual A., G. 2002, A\&A, 381, 524 